Potravinarstvo Slovak Journal of Food Sciences

vol. 14, 2019, p. 76-87

https://doi.org/10.5219/1195

Received: 27 September 2019. Accepted: 26 November 2019.

Available online: 28 February 2020 at www.potravinarstvo.com

(C) 2020 Potravinarstvo Slovak Journal of Food Sciences, License: CC BY 3.0

ISSN 1337-0960 (online)

\title{
INFLUENCE OF REDUCTION ON ADHESIVE PROPERTIES
}

\author{
Igor Stadnyk, Volodymyr Piddubnyi, Svitlana Krsnozhon, Nataliia Antoshkova
}

\begin{abstract}
An analytical analysis of the action of rolls on the medium and its behaviour at adhesion bonds with its surface are carried out. The methods and means of carrying out researches on determination of surface roughness are offered, and the experimental setting for determining the adhesion strength is developed. To reveal the essence and understanding of the general research execution, a number of hypotheses for the determination of adhesion are given and a generalized approach to the definition of adhesion is given. The physical nature of the influence of the roughness of the roller surface on the injection of the dies depends on the shape and angle of roughness, the application of mechanical forces, the degree of its previous dispersion (recipe) and its physical and mechanical properties. The nature of the contact interaction of the dough with the rough surface of the roller working organ in the injection nozzle of the fumigation machine is established. Violation of these mutual relations leads to the production of poor-quality products and a reduction in the efficiency of the machine. The contact area of the adhesive and the component forming work for overcoming the adhesion and deformation of the environment in determining the criteria influencing the process according to each particular period of the deformation stage are substantiated. The obtained data give an answer to a number of questions about the possibility of interaction of the surface of the working bodies from the environment. On the basis of their data, the actual change in the contact adhesion in the roller unit of the molding machine with a comparative analysis of existing ones with the newly designed design is considered. It is established that in order to provide a constant area of actual contact, which contributes to better adhesion, and, accordingly, the passage of a qualitative process of tightening, compression and pouring, the necessary condition is the consistency of the specified criteria. This means that the actual contact area $S_{f . \kappa}$, varies from $S_{N . \kappa}$ to $S_{c}$ depending on the ratio of parameters.
\end{abstract}

Keywords: dough; adhesion; adhesive; substrate; forming channel; deformation; stress

\section{INTRODUCTION}

In food technologies, in the preparation of raw materials, the receipt of semi-finished products, finished products, their storage is important interaction product with various moving and stationary surfaces. Such interaction, as a rule, leads to the adhesion of the product to the surface of the working bodies, working chambers of technological equipment, as well as structural and technological materials etc. In technology, the phenomenon of sticking is called adhesion (Zimon and Yevtushenko, 1985).Adhesion of the food masses is often undesirable. Oftentimes one has to face the phenomenon when the dough is sticking to the surface, and when it is removed, the part remains on the surface of the latter. This results in loss and deterioration of the semi-finished product and its appearance. Consequently, it negatively affects the efficiency of the use of equipment. The quality of the product, leads to increased costs of raw materials and energy resources, complicates the sanitary conditions of enterprises. In the previous part of our research, an example of the effect of adhesion is given. Effect of adhesion in the formation of bagels, drying on a molding machine, where the surface of roller working bodies is rough, has grooves, grooves. This is evidenced by the sticking of the dough to the surface of the roller working bodies of the molding device (Stadnyk et al., 2018). With the wide introduction into food production of modern automated, integrated mechanized lines, when the processing speed of the non-Newtonian food masses has significantly increased and new building materials are widely introduced, there is always a need to study the strength of adhesion, modelling processes. Modelling of technological processes is directed to the separation zone, considering both the type and condition of the surface, and the structural and mechanical properties of the nonNewtonian food masses. Processes associated with the adhesion of structured food masses, such as dough, baking and flour confectionery products, are still poorly studied and especially difficult to model. This phenomenon was investigated by many inventors who revealed the essence and possible ways of decreasing adhesion, its determination with the help of theoretical and experimental studies and was substantiated by mathematical modelling. Therefore, in our opinion, to 
reduce the negative impact of adhesion on technological processes can be by means of a comprehensive study of this phenomenon, i.e., based on the application of modern methods of simulation, analysis of processes in the contact area, the product -product.

\section{ANALYSIS OF LATEST RESEARCHES}

The adhesion of the elastic-plastic food masses is realized at the boundary between the two solids. Elastic-plastic bodies have abnormal viscosity, which varies depending on the shear stress, mass properties and other factors. The reason for the variability of viscosity is the peculiarities of the structure of elastic-plastic bodies. Adhesion as a superficial phenomenon arises at the boundary of the distribution of two phases of heterogeneous condensed bodies: the food masses - one phase, the contact surface the second phase (Moriarty et al., 2011). Superficial properties of the food masses, in particular, adhesion, depend on the bulk properties of the masses themselves. The latter determine the contact area of two bodies, which affects the amount of adhesion and its consequence, which characterizes the condition of the surface after removing the adherent mass. The separation of material from a solid contact surface may have adhesion (the boundary separates through the surface of the contact surface), cohesive (the fringe is contained in the product layer) and mixed (Hoevar et al., 2014). Adhesion is due to various forces and connections by nature, they can be divided into two groups. The first group of forces is manifested in the convergence of two bodies and in the absence of contact between them, when there is a gap of a certain magnitude. The same forces act after the violation of the contact of dissimilar bodies and cannot exist in the absence of contact.As a result of the works under consideration (Stadnyk et al., 2019) it was established that the structural parameters of the rolls are directed to ensure the flow of the dough during its alignment and redistribution in the volume of its mass due to the smooth drag, transport and injection in the gap between them. Although the process of injection of medium (dough) with rollers at first glance seems simple, but the construction of its mathematical model and the search for the basic calculations of dependencies is quite complicated. Now there are a number of solutions for this problem, which are based on simplification of the actual process and do not consider the influence of the elastic-viscous and plastic structure of the dough and the action on it of the pressure fluctuations. Due to the fact that the pressure of the dough on the roller working bodies is transmitted on a normal basis, on the basis of the applied working pressure and the diagrams of its change over the length of the working chamber, one can determine the forces acting on them in the zones of power and pumping. (Stadnyk et al., 2018), grain storage.Surface adhesion is considered to be a complex physical and chemical process that depends on surface properties such as topography, roughness, hydrophobicity, chemical composition and surface energy; the initial amount of the medium, its size, temperature and $\mathrm{pH}$ of the environment, etc. (Hoevar et al., 2014; Whitehead and Verran, 2007; Merritt and Yuehuei, 2000). However, among many of the factors that influence the process of adhesion, researchers (Whitehead and Verran, 2007; Merritt and Yuehuei, 2000) believe that surface properties play a major role. As a result, three theories of microbial adhesion to the surface have been proposed: thermodynamic, DLVO (Deryaguin-Landau-VerweyOverbeek) theory and the theory of extended XDLVO (Hoevar et al., 2014; Whitehead and Verran, 2007). The thermodynamic theory is based on the fact that when the particles are attached to the surface there is a change in the total free energy of Gibbs (the energy, which is determined in the closed system). This energy is calculated by the equation of Lifshitz-van der Waals and the acidic interactions of Lewis (Hoevar et al., 2014).

$$
G_{A D N}=\Delta G_{L W}+\Delta G_{A B}
$$

Where:

$\Delta G_{\mathrm{ADH}}-$ change in the total free energy of Gibbs involved in adhesion; $\Delta G_{\mathrm{LW}}$ - the change in the total free energy of Gibbs and the forces of Lifshitz-van der Waals; $\Delta G_{\mathrm{AB}}-$ change of free energy of acidic main forces of Lewis.

The thermodynamic theory assumes that adhesion is always a reverse and distance-independent process. This theory does not determine the influence of surface charge and the concentration of electrolytes in the environment. It is believed that this theory is most accurate when working with uncharged surfaces or in the presence of a large number of electrolytes in the medium (Hoevar et al., 2014). The theory of DLVO is based on the thermodynamic theory, and also suggests that adhesion is the sum of interphase energies. This theory believes that colloidal particles of a disperse system can easily unite with each other until contact of their liquid diffuse shells occurs.

$$
U^{D L V O}=U^{L W}+U^{E L}
$$

Where:

$U^{\mathrm{DLVO}}$ - full energy interactions; $U^{\mathrm{LW}}-$ the energy of the forces of Lifshitz-van der Waals; $U^{\mathrm{EL}}-$ electrostatic energy interactions.

The theory assumes that adhesion can be reciprocal and depends on distance. It is most accurate when electrostatic forces prevail, but it is limited in the case of ignoring the effect of polar interactions (Hoevar et al., 2014). In order to more accurately model microbial adhesion, the theory of XDLVO, based on the thermodynamic and DLVO theory, was proposed. According to this model, it is assumed that the adhesion is the sum of the forces of Lifshitz-van der Waals, the electrostatic and free energy of the acid-base forces of Lewis.

$$
G_{A D N}=\Delta G_{L W}+\Delta G_{A B}
$$

Where:

$U^{\mathrm{DLVO}}$ - full energy interactions; $U^{\mathrm{LW}}$ - the energy of the forces of Lifshitz-van der Waals; $U^{\mathrm{EL}}$ - electrostatic energy interactions; $U^{\mathrm{AB}}$ - the energy of the acidic main forces of Lewis.

As in the case with the DLVO theory, the XDLVO model believes that adhesion can be reciprocal and depends on distance.However, researchers (Hoevar et al., 2014; Whitehead and Verran, 2007) believe that all three theoretical models, which aim to reveal the essence of adhesion to the surface, are designed for an ideal colloidal system. 


\section{Scientific hypothesis}

In production conditions, adhesion is a much more complicated process and its attachment to the surface may occur in different ways. Therefore, in our view, the process of adhesion to the surface in practice often differs from the above described theories. This is due to the fact that the surfaces of solid materials are exposed to various contacting media, adsorb organic and inorganic substances, thus forming a conditioning layer, to which the attachment of the contact medium comes. In the future, under the action of the driving forces, the formed air conditioning layer changes the physical and chemical properties of the surface, and this affects the process of adhesion.

On the basis of the above, we consider that the adhesion of the medium to solid surfaces is a two-phase process, which consists of the initial inverse (physical) and the next irreversible (molecular or cellular) phase. Adhesion to the solid surface can also be passive or active, which depends on the driving forces and transport of cell media on the basis of gravity, diffusion, or hydrodynamic forces. In addition, the process of adhesion is influenced by the physical and chemical properties of the medium, phase composition and surface roughness.

Therefore, when studying adhesion, we pay attention to the surface roughness and the parameters of the topography. Thus, proceeding from this, the process of adhesion is closely related to the amplitude surface parameter (roughness) and its spatial changes, which are characterized by morphological features of the surface (topography). Therefore, the theory of attachment of the medium to the surface should consider mainly the physical and chemical aspects of the surface of materials, and to a lesser extent, pay attention to the morphological and physiological features of the medium.

\section{MATERIAL AND METHODOLOGY}

A dough with a moisture content of $33 \%$, for high quality wheat flakes on pressed yeast, was prepared in an opaque manner with a fermentation time of 60 minutes at a temperature of $32-33{ }^{\circ} \mathrm{C}$. The quality of the pressed yeast corresponds to the DSTU. Characteristics of wheat flour:

- mass fraction of moisture, $\%-14.5$;

- $\quad$ the content of raw gluten, $\%-28$;

- resistance gluten compression on the device IDK-1, per.pril. - 54;

- gluten stretch, cm-14.

The study of the dough injection process was carried out on the molding machine B-54 of the confectionery factory (Ternopil). It is known from work (Zimon and Yevtushenko, 1985) that the adhesion is determined by separating the medium from the surface, measuring the separation effort. In this effort, the adhesion resistance of the medium is calculated. Therefore, the adhesiveness of the Fvd (equal to the ratio of the fracture effort of the Fvd model to the area of the nominal contact Shk) depends on the size of the surface and adhesion, the conditions of contact and the separation of the dough. Adhesion was seen as a process that occurs in time when the surfaces of two heterogeneous bodies come into contact or are violated. For the quantity that quantitatively evaluates the adhesion, the work of the separation (strength) and the unstable process of filling the medium (dough) of the groove surface of the roll at the pressure of the previous loading $\mathrm{Pk}$ were used.

The problem that complicates the determination of adhesion strength is the establishment of the actual contact area. After all, the size of the area of actual contact is influenced by many factors: normal pressure, the nature of the contacting bodies, as well as external factors temperature, tenseness, duration of the previous load, speed of growth separation effort. These factors have a different effect on the change in the actual contact area.

An analysis of existing methods of studying the adhesion properties of food products showed that all the methods considered have their advantages and disadvantages. In this regard, an experimental device was developed for the study of adhesion properties of the dough consisting of vessel 1 (Figure 1), plate 2, rod 3, cargoes 4, measuring vessel 5 , capacity 6 , flexible hose 7 , valve 8 , a cable 9, a pulley 10 , a tripod 11 , a guide 12 , and a valve 13 .

This installation worked as follows. A bowl of uniform dough was placed in the vessel 1 , on top of which a wide plate 2 with a rigidly fixed rod 3 was installed. The previous loading was carried out by measuring loads 4 which were mounted on the plate 2 . In a measuring vessel 5, a liquid from the container 6 was fed by means of a flexible hose 7 and thus created the separation effort that was transmitted to the plate 2 by means of a rope 9 , which is one end attached to the rod 3 , and the other to a measuring vessel 5. In the vessel 6 , a constant level of liquid was maintained. The valve 8 made it possible to adjust the flow rate of the liquid to the measuring vessel 5 and thus adjust the rate of application of the force to the plate 2 .

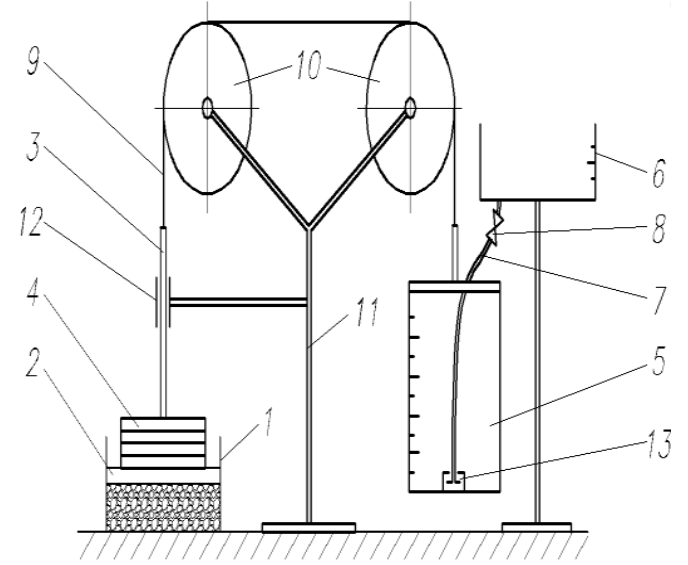

Figure 1 Scheme of the installation for the study of adhesion propertiesprotein dispersed phase:1 a vessel; 2 - plate; 3 - stock; 4 - cargoes; 5 - measuring vessel; 6 - capacity; 7 - flexible hose; 8 - valve: 9 - cable: 10 - pulleys;11 - tripod; 12 - guiding; 13 - valve.

The change in the direction of the force by $180^{\circ}$ was carried out with the help of pulleys 10 which are attached with the possibility of rotation around their axis on the tripod 11. As a previous load so and the separation effort acted at right angles to the plate 2 . 
This was provided by means of a guide 12 rigidly attached to the tripod 11. After the complete separation of the plate, the 2-meter vessel 5 moved down and the valve 13 blocked the feeds in the liquid from the vessel 6 . The separation effort was determined through the volume of the liquid that was in the measuring vessel 5 after the plate was detached.

The developed experimental setup allowed to regulate parameters that influence the adhesion interactions: the pressure of the previous loading $(0-10 \mathrm{kPa})$, the separation effort $(0-100 \mathrm{~N})$, the rate of growth of the separation effort $\left(0.2-2 \mathrm{~N} . \mathrm{s}^{-1}\right)$ and contact area

The quarrying of the experimental set-up was to determine the force to be applied to rod 3 to ensure that the plate 2 is detached from the bottom of the vessel 1 provided there is no layer of the protein disperse phase in vessel 1.

The dough was applied uniformly to the bottom of a rigid vessel with cylindrical walls in such a way that the height of the layer was $1.2 \times 10^{-2} \mathrm{~m}$. The area of the bottom of the vessel corresponded to the area of the plate and amounted to $7.8 \times 10^{-3} \mathrm{~m}^{2}$.

Adhesion strength was determined by substituting data obtained during the experiment into the following formula:

$$
P_{a}=\frac{F-F_{0}}{S_{k}}
$$

Where:

$\mathrm{F}_{0}$ - separation effort in the absence of the test material in the vessel, $\mathrm{H}$.

\section{Methods of study of roughness of a surface}

Experimental researches were carried out using modern technical and standard methods (determination of surface roughness of steel), microscopic (light and electron microscopy of the process of formation and degradation of dough residues), spectrophotometric (optical density (density), mathematical and theoretical modelling, statistical). Used plates of stainless corrosion-resistant nickel-chrome austenitic steel in the size of $30 \times 30 \mathrm{~mm}$ and $5 \mathrm{~mm}$ thick, with roughness of the surface $\mathrm{R}_{\mathrm{a}}=2.687 \pm 0.014$ microns, $\mathrm{R}_{\mathrm{a}}=0.95 \pm 0.092$ microns, shown in Figure. 2. Their roughness corresponded to the surface of the roll.

The roughness of the surfaces of the stainless-steel plates was determined using a profiler of the mark 296. The profile of the profile (Figure 3) includes: a rack 3 for installing parts with a diamond measuring needle, a drive 1 for moving the sensor along the measured surface and an electronic unit 2 for control and calculation roughness of the surfaces. The measured part is mounted on the plate of the rack 3 . If the part has a cylindrical shape, the prism 5 is installed on the stove plate and the measured part is mounted on the prism 5. The part is set so that the measured surface is perpendicular to the measuring plane. The nut 6 is designed to move the sensor 4 with actuator 1 along the guide rack 3 and install the diamond needle of the sensor 4 on the measured surface of the part.
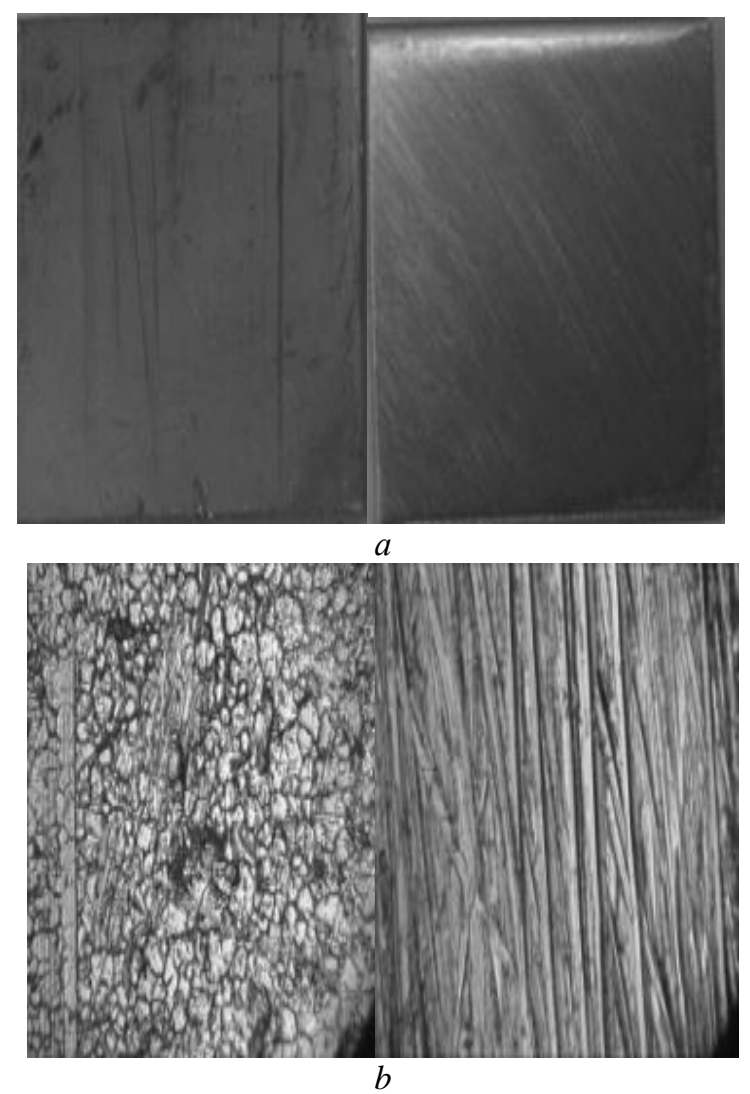

$\mathrm{R}_{\mathrm{a}}=(2.68 \pm 0.014)$ microns, $\mathrm{R}_{\mathrm{a}}=(0.95 \pm 0.092)$ microns, $\mathrm{R}_{\mathrm{a}}=(0.63 \pm 0.087)$ microns

Figure 2 The appearance of the stainless plates with different roughness: a- native appearance of the plates; B-appearance of plates using microinterferometer MII4U4.2 (increase 1500 times).

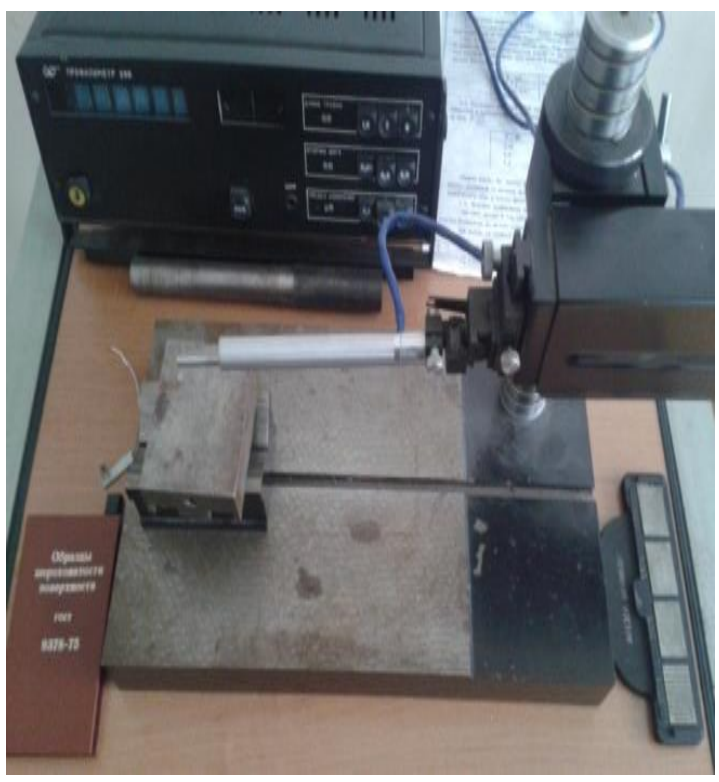

Figure 3 Ploofilometer mark 296. 
Electronic block 2 is executed in a desktop version. On the block front panel there are: a digital scoreboard for the measured $\mathrm{Ra}$ value; indicator of the working area; power button.

\section{Statistical analysis}

Considering the chaotic interaction of the dough during its displacement, where the change of interaction occurs between the broad-walled surface in the surface of the roll, the task planning experiment with the use of a full factor experiment of the second order is compiled. With two factors, the model of the experiment's function has the form:

$$
y=f\left(X_{1} X_{2}\right)
$$

According to the results of the experiment, we obtain a regression equation of the second order.

$$
Y=b_{0}+b_{1} x_{1}+b_{2} x_{2}+b_{11} x_{1}^{2}+b_{22} x_{2}^{2}+b_{12} x_{1} x_{2}
$$

For experiments a plan with corresponding matrices of experiment planning with the number of experiments and boundaries of factor changes has been drawn up. The matrix is a list of options taken in this series of experiments. Independent variables were selected from the analysis of the nature of the effect on the change in the contact angle of the dough with the roll (forums number 5). As a parameter of optimization, the side a and the contact area of the broadened surface $S$ are used respectively.

$\mathrm{X}_{1}$ - side length a, mm;

$\mathrm{X}_{2}$ - the contact area of the broad surface $\mathbf{S}, \mathrm{mm}^{2}$. Experiments were carried out on the basis of mathematical planning. Determining which factors influence the change in the angle of contact, we determine their level variations and the step of variation. The main factors and their variation equation are given in Table 1.

Output parameters were:

$\mathrm{y}_{1}$ - change in the angle of interaction of the dough in height of its movement in the gap between the burrs. The displacement height of the test mass was recorded by the visual control method on a scale applied to the working roller;

$\mathrm{y}_{2}$ - change the angle of interaction of the dough on its contact area on the roll surface. The weight of the test was fixed by the method of visual inspection according to the photographs.

Using the obtained data of the regression coefficients, we make a regression equation for $\mathrm{y}_{1}$ and $\mathrm{y}_{2}$

$$
\begin{gathered}
y_{1}=-54.39-3 a+12.07 S++0.59 a^{2}+0.21 a S \\
-0.33 S^{2} \\
y_{2}=-17.43+0.8 a+3.13 S+0.11 a^{2}-0.09 a S \\
-9.9 S^{2}
\end{gathered}
$$

The analysis of the impact of the surface roughness of the roll confirms our opinion about the activity of adhesion at the injection stage and its dependence on the driving forces. From the graphical dependencies of Figure 5-8 it is quite clear about the influence of the parameters a and $S$ on the process flow. Therefore, the change in the angle of interaction of the dough in its height of movement (Figure 5) depends significantly on $\mathrm{X}_{2}-$ contact area and quite significantly depending on $\mathrm{X}_{1}$ - the length of side a (the basis of latitude).

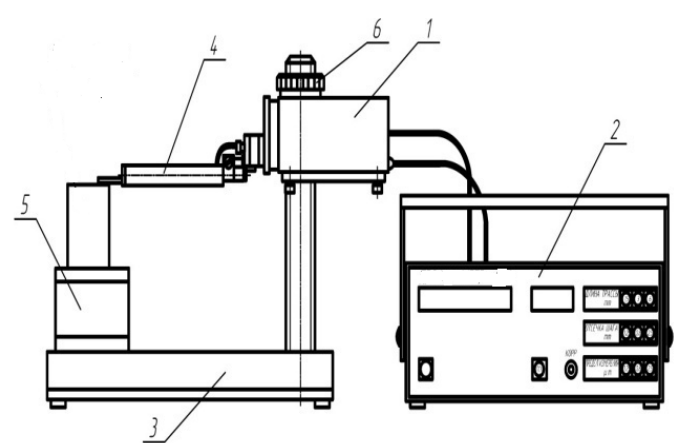

Figure 4 General view of the profile mod. 296: drive -1 ; block electronic -2 ; rack -3 ; sensor -4 ; prism -5 ; nut -6 .

Table 1 The main factors and their variation equation.

\begin{tabular}{lcc}
\hline & $\begin{array}{c}\text { Variable } \\
\text { factors }\end{array}$ & $\begin{array}{c}\text { Variable } \\
\text { factors }\end{array}$ \\
\cline { 2 - 3 } $\begin{array}{c}\text { Characteristics of the } \\
\text { plan }\end{array}$ & $\begin{array}{c}\text { the area of } \\
\text { contact of the } \\
\text { broader } \\
\text { side length a } \\
\mathrm{X}_{1}, \mathrm{~mm}\end{array}$ & $\begin{array}{c}\text { surface } \mathbf{S} \\
\mathrm{X}_{2}, \mathrm{~mm}^{2}\end{array}$ \\
\hline Basic level, $\mathrm{X}_{1}^{(0)}$ & 4.5 & 12 \\
The step of variation & 1.5 & 3 \\
Lower level $\mathrm{X}_{1}^{(-)}(-1)$ & 3 & 9 \\
Upper level, $\mathrm{X}_{\mathrm{i}}^{(+)}(+1)$ & 6 & 16 \\
\hline
\end{tabular}

Table 2 The experiment plan and its results.

\begin{tabular}{cccc}
\hline $\mathrm{X}_{1}(\mathrm{a}, \mathrm{mm})$ & $\mathrm{X}_{2}\left(\mathrm{~S}, \mathrm{~mm}^{2}\right)$ & $\mathrm{y}_{1}$ & $\mathrm{y}_{2}$ \\
3 & 9 & 30 & 5.6 \\
4.5 & 9 & 35 & 7 \\
6 & 9 & 40 & 9 \\
3 & 12 & 45 & 9.8 \\
4.5 & 12 & 50 & 10 \\
6 & 12 & 64 & 10.8 \\
3 & 16 & 60 & 11 \\
4.5 & 16 & 68 & 11.6 \\
6 & 16 & 75 & 12.4 \\
\hline
\end{tabular}

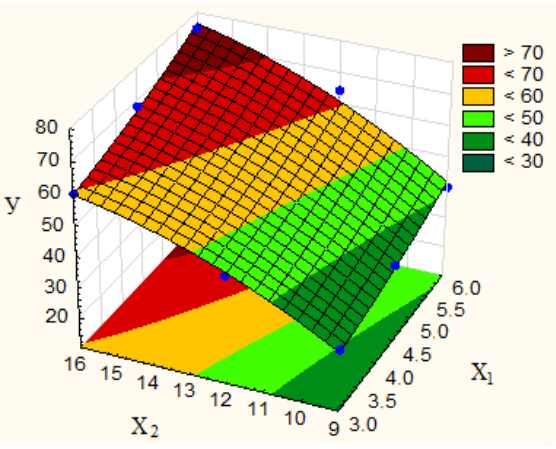

Figure5 Two-dimensional section of the surface of the response as a function. 
The optimum values are within the range of $50-600$ at the values a $=4.5-5.5 \mathrm{~mm}, \mathrm{~S}=11-13.5 \mathrm{~mm}^{2}$. To change the angle of interaction of the dough along its contact plane $\mathrm{y}_{2}-$ the optimal values are at the corner $6-9^{0}$ at values a $=5-6 \mathrm{~mm}, \mathrm{~S}=10.5-12.5 \mathrm{~mm}^{2}$.

Thus, the surface of the roll really creates conditions for the movement of the mass of the dough both on its surface and in the layers placed in the working chamber.

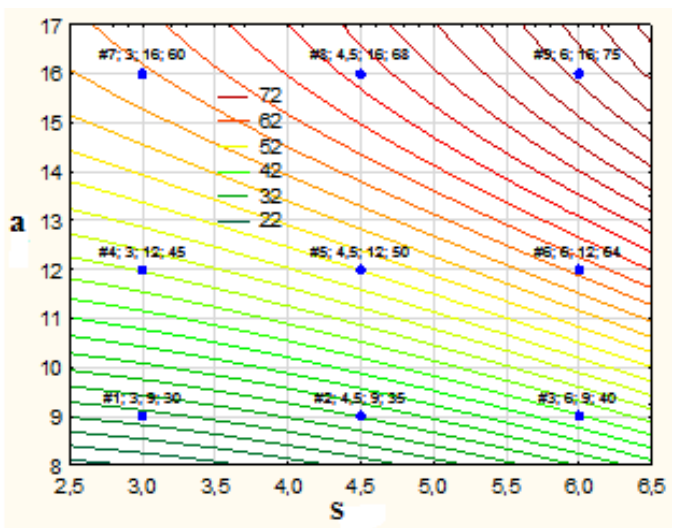

Figure6 Surface response $\mathrm{y}_{1}=\mathrm{f}(\mathrm{a}, \mathrm{S})$.

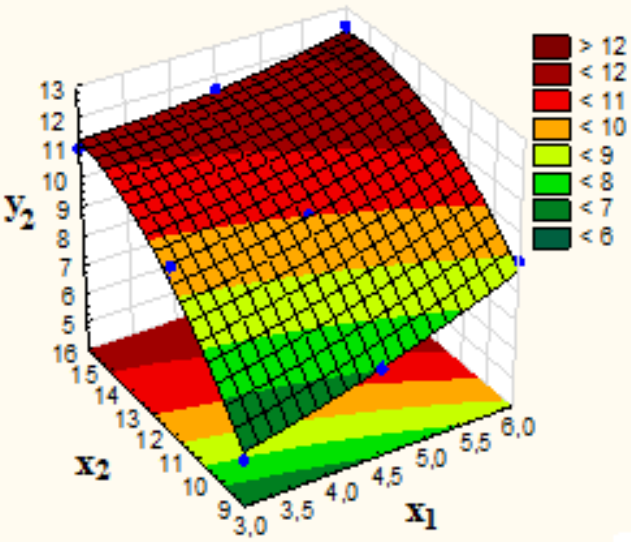

Figure 7 Two-dimensional section of the surface of the response as a function $\mathrm{y}_{2}=\mathrm{f}(\mathrm{a}, \mathrm{S})$.

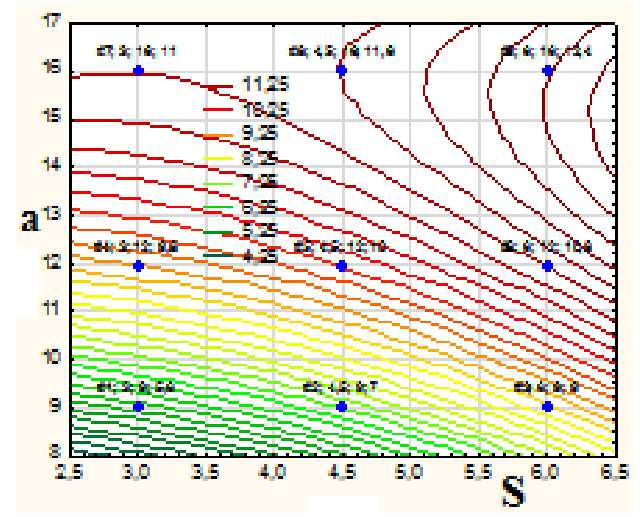

Figure 8 Surface response $\mathrm{y}_{2}=\mathrm{f}(\mathrm{a}, \mathrm{S})$.

\section{RESULTS AND DISCUSSION}

The formed adhesion on the surface of the roll consists of an inhomogeneous structure, resulting in a gas concentration gradient, in particular, reducing the amount of oxygen from the periphery to the depth of the environment, $\mathrm{pH}$ gradients and temperature. Such gradients of functioning provide contact between the two bodies, resulting in phenotypic resistance to abrupt change in environmental factors. Data from the scientific literature (Hoevar et al., 2014 Stadnyk et al., 2016; Gorbatov, 1979) indicate that when studying adhesion, it is necessary to approach complexly and consider, along with roughness, the topography of the surface, as these quantities are interdependent. Consequently, the adhesion process is closely related to the amplitude parameters of the surface (roughness) and its spatial changes, which are characterized by morphological features of the surface (topography).

Studies on the effect of terrain and surface roughness on adhesion are not straightforward. Thus, according to (Stadnyk et al., 2018; Nikolaev, 1976; Jullien et al., 2003), there is a correlation between surface roughness and adhesion, with the attachment of the medium to the surface increasing with increasing roughness. However, other studies indicate (Kolari, 2003; Kukhtyn et al., 2017; Langsrud et al., 2016) that there is practically no correlation bond between the roughness of the surface of the stainless steel and the roughness range of 0.01 to $3.3 \mu \mathrm{m}$. It has also been found that adhesion on surfaces with high roughness reduces the efficiency of heat transfer in heat exchangers by about $15 \%$ (Kukhtyn et al., 2017).

Therefore, the conflicting data obtained by scientists regarding the effect of the surface roughness of stainless steel on the adhesion process are obviously related to the experiments under different conditions using different media, materials and methods of study. However, scientists have concluded that such elements of the surface topography as scratches, cracks, holes, protrusions, cracks play an important role in the adhesion process (Kolari, 2003; Monds and O'Toole, 2009; Moons and Michiels, 2009; Zogaj et al., 2003).

The results of the analytical review and the conducted research and statistical modelling made it possible to approach the development of methods for determining the surface and adhesion strength by mathematical modelling. Based on the above and the data processing, two mathematical modelling approaches are proposed that can be used to determine the adhesion strength and strength.

\section{Method of determining the wide surface of the roll}

The results of the computational experiments allowed to investigate the effect of changing the angle of roughness of the roll surface on the interaction with the dough. From the studies it is clearly seen that the angle of roughness of the surface when interacting with the dough affects the adhesion properties. This approach allows us to determine rational design parameters that will contribute to the intensification of the dough injection process. Based on previous studies (Stadnyk et al., 2019), when studying adhesion, it is necessary to approach the complex and consider, along with the roughness, the topography of the surface, since these values are interdependent. The surface 
roughness refers to the two-dimensional surface of the material and is usually described as arithmetic mean roughness $(\mathrm{Ra})$ and average square roughness $(\mathrm{Rq})$. At the same time, the topography has three dimensional parameters and describes the elements of the shape of the surface. Under the strength of adhesion, adhesion must be understood as a function of factors such as separation speed, duration and pressure of the previous contact, flat contact, etc. It is necessary to distinguish between the strength of adhesion, which is measured in $\mathrm{H}$ (Newton), and the strength of adhesion $-\mathrm{N} . \mathrm{m}^{-1}$.

Interaction on the boundary of two phases, that is, the dough and the surface of the rolls, occurs from the first seconds of the molding machine. Therefore, the phenomenon of wetting the roller surface is related to the ratio of surface tensions $(\sigma)$ of the adhesive and substrate. To achieve wetting on the surface of a well-adhesive roller, it must be ensured that the surface tension of the substrate is greater than the surface tension of the adhesive. This will accelerate the process as a whole with a corresponding reduction in energy costs.

The studies carried out by the authors found that the strength of the adhesion of the dough at speeds of its separation from the roller working body of the molding machine was not determined more than $1 \mathrm{~m} . \mathrm{s}^{-1}$. This is due, first of all, to the lack of simulation of the existing processes of formation, transport of the dough, which is mainly due to relatively small speeds of movement of the working bodies of the molding machine.

Power interaction of the medium with a roll occurs on its surfaces after the discrete injection of the mass of the test. Since the method of formation and the shape of the interaction profiles of the dough with the surface of the roll has a significant impact on the quality characteristics and the injection process, a number of comparative studies have been carried out to determine the rational roughness section of the roll.

To increase the force of adhesion between the viscous medium and the leading roller working body is possible by increasing the angle of coverage in accordance with the well-known law of Euler:

Where:

$$
S_{n a b}=S_{n a g} e^{\alpha f}
$$

$S_{\text {nab }}$ and $S_{\text {nag }}$ - respectively, tightening at the points of the runoff of the medium and its coincidence with a rough surface; $\alpha$ - the angle of coverage of the medium; $\mathrm{f}$ - coefficient of friction in a pair of materials.

The change in the angle of coverage of the medium is achieved due to the geometric orientation of the tightening and its injection. The problem of geometric synthesis of the system with an increase in the angle of coverage of the medium is solved on the basis of geometric bonds. Consider cases of power interaction, in which the line of vertices of roughness of a roll:- parallel to the vector of injection velocity $v_{\mathrm{p}}$ and the circular power $\mathrm{R}_{\mathrm{KB}}$ (angle $\alpha=0^{\circ}$ );

- not parallel to the velocity vector injection vr and circular power $\mathrm{R}_{\mathrm{KB}}\left(\right.$ angle $\left.\alpha>0^{\circ}\right)$.

The angle $\alpha$ depends on the lifting of roughness (Figure 6). Roll movement in the middle during its injection can be divided into 3 components:
- rectilinear horizontal in the direction from the axis of the roll to the inner surface of its body, due to centrifugal force Pvb;

- rotational horizontal in the direction of rotation of the roll due to the force of the knee $P_{K B}$;

- straight vertical from the force of gravity on the dough layer of the dough that is above it $P_{T G}$.

Due to these movements, the friction forces (adhesion) appear on the upper and lower front roughness of the roller surface. The three components of the dice movement described above correspond to the coordinate axes of the XYZ Cartesian coordinate system (Figure 9). That is, the direction of the axis OX (vertical axis) coincides with the direction of gravity, $P_{T G}$. OY (horizontal axis) with the direction of action of the centrifugal force $P_{V B}, \mathrm{OZ}$ (orthogonal) with directional velocity $\mathrm{v}_{\mathrm{KB}}$, circular force $P_{K B}$ and the forces of compression of the dough $P_{C T}$. With a steady state of work, we assume that the velocity of the circle $v_{\mathrm{KB}}$ will be equal to the pumping speed $v p$ $\left(v_{K B}=v_{H}\right)$.

In the case of $\alpha=0^{\circ}$, the vertical coordinate plane XOY will be perpendicular to a plane passing through lines of roughness to its front surface. If $\alpha>0{ }^{\circ}$, then the angle between the above-mentioned planes will be $90^{\circ} \pm \alpha$.

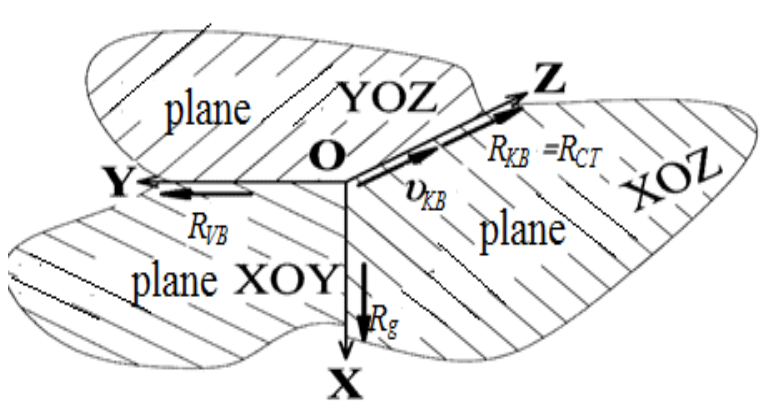

Figure 9 Coordinate system, which considered power interaction: $\mathrm{R}_{\mathrm{VB}}-$ centrifugal force; $\mathrm{Rg}$ - gravity force; $\mathrm{R}_{\mathrm{KB}}$ - the force acting on the dough; $\mathrm{P}_{\mathrm{CT}}$ is a compressive force; $v_{\mathrm{KB}}-$ circular speed.

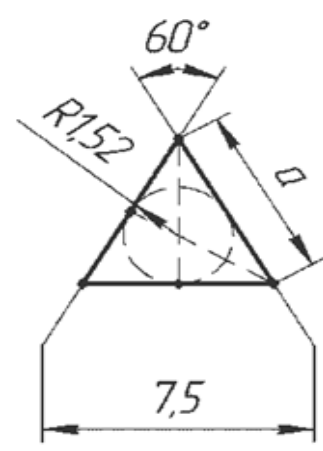

a)

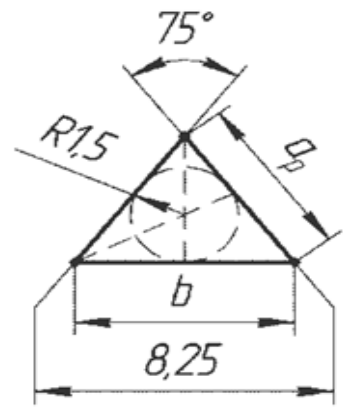

b)
Figure 10 Cross-sections of roughness of different profiles with an area of $1.2 \mathrm{~mm}^{2}$ each: a) triangular, equilateral; b) triangular, equidistant.

For comparison, two triangles were chosen (an equilateral triangle, an equilateral triangle with an angle at 
the vertex of 75 , cross-sections of roughness with an area of $1.2 \mathrm{~mm}^{2}$ each (Figure 10).

For an equilateral triangle $\mathrm{S}$, the length of a side is determined by the formula:

$$
a=\sqrt{S \frac{\sqrt{3}}{4}}
$$

For an isosceles triangle, the area $(\mathrm{S})$ will be determined by the formula:

$$
S=\frac{1}{2} a_{p} b \sin \alpha
$$

Where: $\mathrm{a}$ - side of the triangle; $\mathrm{b}$ - the basis of the triang $\{\mathrm{e} ; \alpha-$ is the angle between the side and the base (for an isosceles triangle with an angle at the apex $75^{\circ} \alpha=52.5^{\circ}$ ).

We will define the projection theorem $\boldsymbol{b}$ :

$$
b=2 a_{p} \cos \alpha
$$

Substituting equation (5) into formula (4) and expressing $\boldsymbol{a}$, we obtain it:

Where:

$$
a_{p}=\sqrt{\frac{S}{\cos \alpha \sin \alpha}}
$$

$S=1.2 \mathrm{~mm}^{2} \rightarrow a p=0.498 \mathrm{~mm}, b=0.606 \mathrm{~mm}$.

\section{Method of determining adhesion strength}

At the moving surface of the rotary rollers, a general work is performed which consists of elastic forces and changes in the contact of the rolling dough: $A_{z}=A_{p r}+A_{k}$.

Work of elastic forces:

$$
A_{p r}=S_{n k}\left(l_{1}-l_{0}\right)
$$

Specific work $1 \mathrm{~kg}$ of dough (in J) can be calculated by the formula:

$$
A_{p r}=\frac{A_{z}}{m_{s r} 10^{3}}
$$

Where: $m_{s r}$ - average weight of dough between surfaces of rotating rolls, $\mathrm{kg}$ :

$m_{S r}=\frac{V}{v}$

Where: $v$ - specific volume; $\mathrm{V}^{-}$camera volume.

The work that is spent on changing the contact of a moving layer of the medium with the surface of the working chamber and rollers to overcome the adhesion and deformation of the medium $A_{d}$, will be:

$$
A_{V}=A_{a d}+A_{d}=F_{V} d x=F_{a d} d h+A_{d} d h
$$

Where:

$F_{a d}, A_{d}$ - the efforts of adhesion and deformation;

$\mathrm{h}-$ the thickness of the medium on the surface of the roll when the separation of its layer on the subsequent process -formation;
$\mathrm{F}_{\mathrm{vid}}$ - the effort of separating a piece of medium from the surface of the roll.

Consider the components forming the work of the separation:

$$
F_{a d}=\int_{o}^{i} f r l d=f_{a d} r l^{2}(6)
$$

Deformation of the environment is determined:

$$
F_{d}=\tau_{0} \frac{V_{v}}{h} r l
$$

Where:

$V_{v}$-the speed of separation of the medium from the surface of the roll under the action of external forces; $\tau_{0}$ - tangential stresses; $\eta$ - plastic viscosity of the medium; $r$ - dough layer on roller; 1 - the length of contact of a part of the working body.

Given the length of the contact area, we obtain the expression:

$$
F_{d}=\tau_{0} \frac{V_{v}}{h} r l
$$

Work determined by the separation effort is spent on overcoming adhesion $\mathrm{F}_{\mathrm{ad}}$ and deformation of the medium, when exiting through a rectangular molding surface between rotating rolls $F_{\text {def }}$

$$
F_{V}=F_{a d}+F_{d}(8)
$$

When studying the process of dough injection, one of its conditions was to change the gap between the rolls and the angular velocity of their rotation. Knowing the mass of the dough, the area of its contact with the surface of the mixing drum, with the help of the proposed method and computer symbolic mathematics, the strength of adhesion is determined.

Initial speeds are selected at three values of the crack ( $\delta=20 \mathrm{~mm} ; \delta=25 \mathrm{~mm} ; \delta=30 \mathrm{~mm}$ ). Trajectory of the mass of the test for three cases in the car chamber: $v_{0}=0.18 \mathrm{~m} . \mathrm{s}^{-1} ; v_{0}=0.32 \mathrm{~m} . \mathrm{s}^{-1} ; v_{0}=0.4 \mathrm{~m} . \mathrm{s}^{-1}$. On the dash movement there is an impedance which is expressed by the coefficient of resistance K. For the three clearances we make the equation of spline approximation:

$$
\begin{gathered}
v_{0}=0.18 m s^{-1} \\
K=4.58 x^{2}-3.25 x+2.7 \\
v_{0}=0.32 m s^{-1} \\
K=3.65 x^{2}-2.9 x+2.69 \\
v_{0}=0.4 m s^{-1} \\
K=2.29 x^{2}-2.26 x+2.73
\end{gathered}
$$

\section{Where:}

$v_{0}-$ speed of the dough movement at the output of the rollers, with different values of the gap;

$\mathrm{K}$ - factor of resistance. 
The graph of the relationship between the coefficient of resistance $K$ and the length of the dough movement at the output of the rollers (x) is shown in Figure 11.

Experimentally determining the value of length (x), substituting it into an approximation equation, one can determine the coefficient of resistance $\mathrm{K}$, which is the sum:

$$
K=K_{\Pi}+K_{a d}
$$

Where:

$K_{n}$ - component of the resistance coefficient considering the air resistance;

$K_{a d}$ - part of adhesion.

The air resistance coefficient is 0.11 .

Consequently, considering the trajectory of the dough movement at $\mathrm{m}_{\mathrm{s}} \mathrm{s}^{-1}$ the most favourable (gap of $30 \mathrm{~mm}$ ), we will substitute $\mathrm{x}=0.3$ into the approximation equation: $K=2.29(0.3)^{2}-2.26 \cdot 0.3+2.73=2.26$

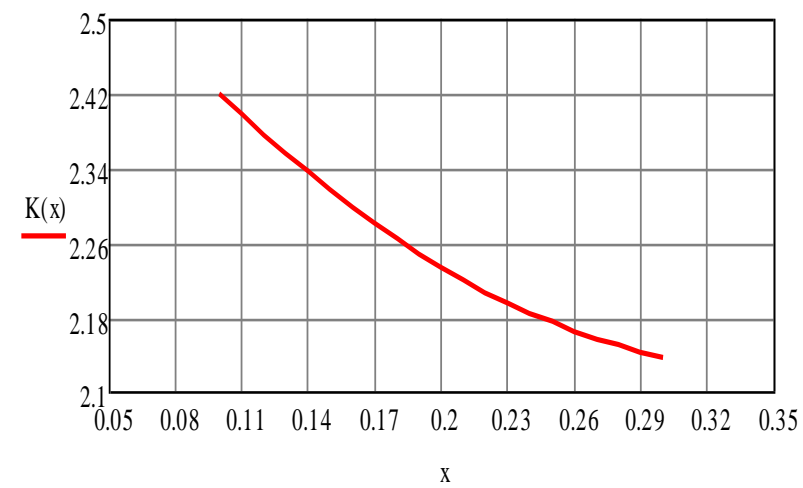

Figure11 Charts of functions K (x) for an initial speed of $0.18 \mathrm{~m} \cdot \mathrm{s}^{-1}$.

We find the coefficient of adhesion, which in our case will be:

$$
K_{a d}=K-K_{n}=2.26-0.11=2.15 \frac{H c}{m}
$$

In accordance with the chosen model of motion, we consider that due to adhesion, the strength of resistance appeared, directed against the movement of the mass of the test, equal to:

$$
P_{o n}=K_{a d} \frac{\partial x}{\partial t}
$$

Since $\mathrm{x}$-axis is chosen for calculations, then:

$$
\frac{\partial x}{\partial t}=v_{0} \cdot \cos \alpha
$$

Substituting this equation in (в), we obtain:

$$
P_{\text {on }}=K_{a} v_{0} \cos \alpha
$$

Knowing the magnitude $K_{a d}, v_{0}, \cos \alpha$, we will define:

$$
P_{\text {on }}=2.15 \cdot 0.4 \cdot \cos 15^{0}=0.831 \mathrm{H}
$$

Where:

$\cos 15$ - the angle between the surface of the roll and the detached moving dough during the injection process.

Area of the nominal contact

$$
S=2 \pi R \frac{1}{8}\left(2 b+\frac{2}{5} \mathrm{R}\right)=2 \pi 0.11 \frac{1}{8}\left(2.02+\frac{2}{5} 0.11\right)=
$$$$
9.0343 m^{2}
$$

Where:

$\mathrm{R}$ - roll radius;

$\mathrm{b}$ - roller width (Figure 12).

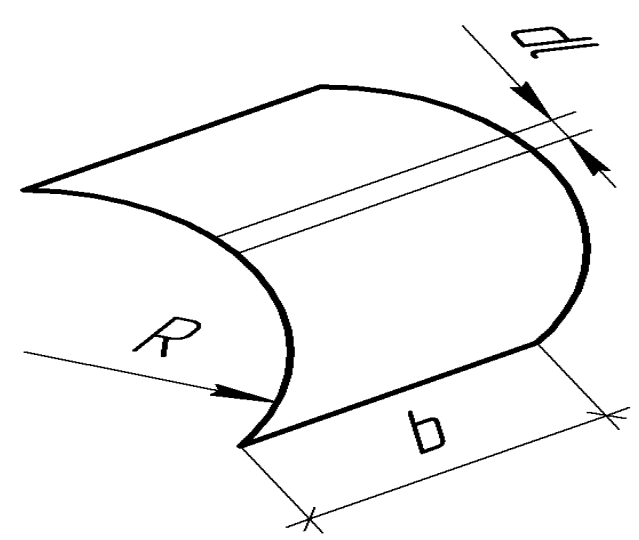

Figure 12 Scheme of Roll.

$$
F_{a d}=\frac{P_{a d}}{S}=\frac{0.831 H}{0.0343 m^{2}}=24.2 \frac{H}{m^{2}}
$$

considering relations (6) and (7) - (8), the separation effort will be in the form:

$f_{a d}+\tau_{0} \frac{V_{v}}{h} F_{V}=S_{r e} \cdot f_{a d}+\tau_{0} \frac{V_{v}}{h}$

With these conditions, it is possible to determine the actual adhesion by the results of an adhesion test at insignificant discontinuation rates when $\mathrm{Vv} \rightarrow 0$.

These conditions are fully consistent with the processes taking place at the pumping stage. In addition, we know the actual contact area of the phases. Minimum actual contact area $S_{\mathrm{nk}}$, formed by a contact of a dough with a roll surface, where $\alpha=1$. The maximum contact area is equal to the surface area of the roll (substrate) Sc (Figure 13).

The nominal contact area is easily determined by the geometry of the adhesive or substrate. The area of the substrate, considering the relief surface (depends on the frequency and type of its processing, Figure 13, type A) is known to us. Therefore, in the general form, the relief of the surface with the side a can be written by a double Fourier series:

$Z=\sum_{m, n-1}^{\infty} a_{m, n} \cdot \sin \frac{\pi m x}{a} \cdot \cos \frac{\pi R Y}{a}$

Where:

$\mathrm{Z}$ - the value of the height of the inequalities;

$a_{r s}$ - Fourier coefficients;

$\mathrm{x}, \mathrm{y}$ - Cartesian coordinates;

$\mathrm{m}, \mathrm{n}-$ Harmonic numbers.

The forces of adhesion in these cases are very small.

Expression (10) characterizes the profile of any surface.

The element is selected on the surface of the areas :

$$
d s=\sqrt{1}+\left(\frac{d z}{d x}\right)^{2}+\sqrt{1}+\left(\frac{d z}{d y}\right)^{2} d x \cdot d y
$$




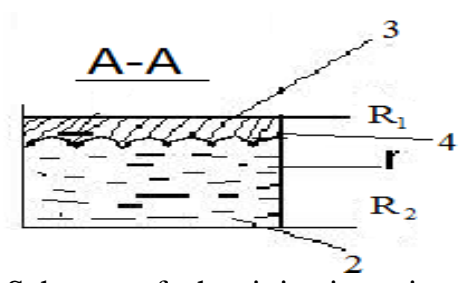

Figure13 Scheme of the injection site: 1-working chamber, 2-dough, 3-rolls; A-determination of the actual contact area of the roller working body and the dough: 2-adhesive (dough); 3 - substrate; 4 - surface of the substrate.

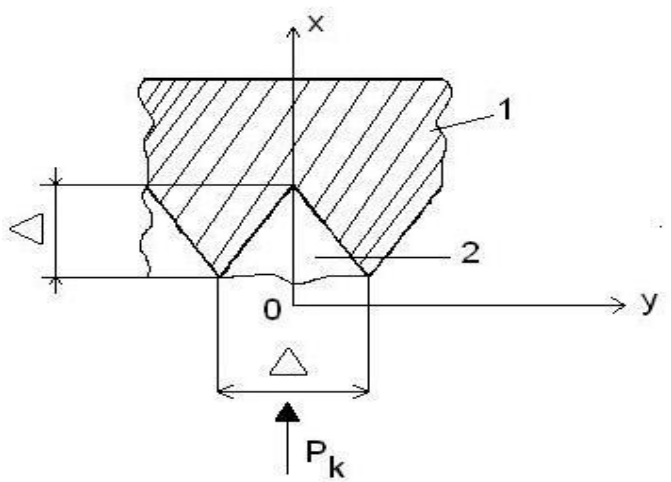

Figure14 Filling the cavity on the substrate surface 1 with adhesive 2 .

Knowing the profile form, i.e. $d z / d x$ and $d z / d y$, it is possible by means of integration to define Sc based on the topography of the surface. So, as the relief of the surface of the roller working body has grooves with the appropriate angle, the angle of the vertex between the groove protrusion $\gamma=60^{\circ}$ at the top of the trapeze. In any arbitrarily selected slit, the relief has the form of an equilateral trapezoid (Figure 16). Then the length of the profile formed by the surface in 2 times will increase the length of the middle line of the profile. Accordingly, the surface area of the substrate will be 2 times greater than the nominal contact area.

In real conditions, the dough does not fully contact the surface of the roller working body. According to the work (Nikolaev, 1976), the filling of the rough surface is proportional to the pressure and time of contact of the dough, as well as its viscosity:

$$
\frac{h}{d} \sim\left(\frac{p_{k} t_{k}}{\mu}\right)^{\frac{1}{2}}
$$

It can be assumed that the dough is in contact with a broad-walled surface at a contact pressure $\mathrm{Pk}$. The width of the roll is characterized by the mean square value of the inequalities Rz. In this case, the cavity on the surface of the roll is filled with a dough. If the size of the macromolecules of the dough is much smaller than the slit of the surface, the law of the mechanics of continuous media propagates in the car's current and the twodimensional flow motion passes.

In this case, the forces of inertia are small, and the forces of hydrostatic pressure, viscous friction and capillary are mutually balanced. In such conditions, to obtain the basic criteria for the similarity of the current, it is sufficient to consider the one-dimensional equation of the dough movement. In isothermal flow, when the values of the temperature of the dough and rolls are equal (this is evident from the experiments of (Stadnyk et al., 2019), the equation of motion has the form:

$Q=-\frac{\partial p}{\partial x}-\frac{1}{\rho} \cdot \frac{\partial \tau_{x y}}{\partial y}$

Where:

$\mathrm{p}$ - pressure;

$\mathrm{x}, \mathrm{y}-$ Cartesian coordinates;

$\tau_{x, y}$-tangential tension;

$\rho$ - density of the dough.

On the free surface of the dough, the conditions of continuity of the normal and the absence of tangential stresses are fulfilled.

We denote the radius of curvature of the adhesive on the surface of the substrate (Figure 14) $\Delta$, then the condition of the continuity of normal stresses on the Laplace formula has the form:

$$
\tau_{x}=\frac{\delta_{r n}}{\Delta}
$$

Where:

$\delta_{r n}-$ surface tension on the boundary adhesive - surface.

The rheological properties of the dough in the region of small deformation velocities, namely, when passing through the gap between rotating roller working bodies, is characterized by the Shvedov-Bingam equation (Stadnyk et al., 2016; Believ, Egorenkov and Pleskachevsky, 1971),

$\tau=\tau_{0} \cdot \sin j=\mu_{p l}$

Where:

$\tau_{0}-$ conditional yield curve;

$\mu_{p l}$ - plastic viscosity;

$\mathrm{j}$ - gradient of strain rate.

The tensile stress in the $\mathrm{xx}$ direction is determined accordingly

$\tau_{x x}=2 B \frac{\partial v}{\partial x}$

Where:

$$
B=\tau \cdot A-\mu_{p l}
$$

$\mathrm{A}-$ the second invariant of the strain rate tensor, obtained for a one-dimensional flow from the ratio:

$A=\frac{\partial v}{\partial x}$

Considering (13) - (15) and equation (11) and boundary conditions (12) can be written as:

$\frac{\partial p}{\partial x}+\frac{\partial}{\partial y}\left(\tau_{0}+\mu_{p l} \frac{\partial v}{\partial x}\right)=0$

$2\left(\tau_{0}+\mu_{P L} \cdot \frac{\partial V}{\partial x}\right)=\frac{\delta_{a b}}{\Delta}$

Replacing differentials with characteristic values

$$
P \approx P_{K} ; \mathrm{Y}-\Delta ; \mathrm{X} \approx \Delta ; v \approx \frac{\Delta}{t}
$$

we get dimensionless components:

$N_{1}=\frac{\delta_{A B} t_{k}}{\Delta \mu_{p l}} ; N_{2}=\frac{\tau_{0 \cdot t_{k}}}{\mu_{p l}} ; N_{3}=\frac{p_{k} t_{k}}{\mu_{p l}}$ 


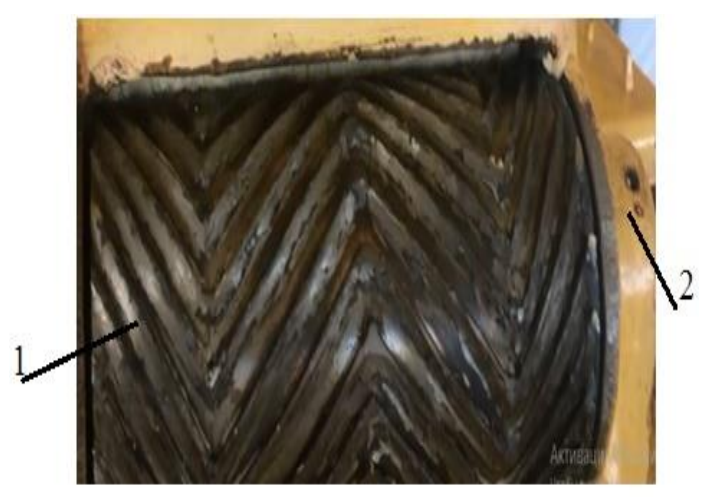

Figure 15 Photo of adhesion of the dough to the surface of the roll of a new design: 1 - roll with screw grooves; 2 - car body.

Criterion $N_{1}$, characterizes the ratio of forces of surface tension and viscous friction; $N_{2}$ analogue of SaintVenant's criterion for conditions of non-stationary movement (flow); $N_{3}$ - takes into account the influence of contact pressure and viscous friction. Thus, completeness complements the grooves of the working body $S_{f . \kappa}$ varies from $S_{N . \kappa}$ to $S_{c}$ depending on the ratio of the parameters of the criteria $N_{1}, N_{2}, N_{3}$. These criteria, as well as a number of others, affect the injection according to each particular period of the corresponding stage of the process. From the criterion equations, it is evident that the plastic viscosity of the dough is of great importance. At the first minutes of the process, the contacts of the medium with the surface of the roller working body pass through the basic principle of slow plastic deformation. With increased contact time with the contact pressure present, adhesion elasticity increases, mainly due to the plastic flow and is determined by the value of plastic viscosity. At the exit from the molding channel formed by the roll's criterion $N_{3}$ loses its meaning and there is a qualitative separation of the given mass of the test to the molding device. At the same time, there are two criteria $N_{1}$ and $N_{2}$ continue to affect the bulk of the dough that is on the roll, retaining its plastic properties and thereby ensuring the cleanliness of the surface (Figure 15).

\section{CONCLUSION}

To ensure a constant area of actual contact, which contributes to better adhesion and, accordingly, the passage of a qualitative process of tightening, compression and pouring, the necessary condition is the consistency of the criteria (18). This means that the actual contact area $S_{f . \kappa}$, varies from $S_{N . \kappa}$ to $S_{c}$ depending on the ratio of parameters. Therefore, to meet the requirements of the process, the gap between the rotary rolls is set at the appropriate distance to create a forming channel, which corresponds to theoretical and practical calculations. In such conditions, qualitative stages of the process in the injection site pass through large stresses of deformations. These conditions must last for a certain period of injection. Therefore, the effect of adhesion should be minimal. It is possible to change the forces of adhesive bonding only if the thickness of the dough layer is sufficiently small and in the separation from the surface of the shafts should be clean.

\section{REFERENCES}

Believ, V., Egorenkov, N., Pleskachevsky, Y. 1971. Adhesion of polymers to metals. Science and Technology, $286 \mathrm{p}$.

Gorbatov, A. 1979. Rheology of meat and dairy products. Russia, Moscow : Pishchevaya Promyshlennost, 382 p.

Hoevar, M., Jenko, M., Godec, M., Drobne, D. 2014. An overviev of the influence of stainless-steel surface properties on bacterial adhesion. Materials and technology, vol. 48, no. 5, p. 609-617.

Jullien, C., Bénézech, T., Carpentier, B., Lebret, V., Faille, C. 2003. Identification of surface characteristics relevant to the hygienic status of stainless steel for the food industry. $J$. Food Eng., vol. 56, no. 1, p. 77-87. https://doi.org/10.1016/S0260-8774(02)00150-4

Kolari, M. 2003.Attachment mechanisms and properties of bacterial biofilms on nonliving surfaces: dissertation theses. Finland : University of Helsinki. 79 p. ISBN 952-10-0345-6.

Kukhtyn, M., Berhilevych, O., Kravcheniuk, K., Shynkaruk, O., Horyuk, Y., Semaniuk, N. 2017. Formation of biofilms on dairy equipment and the influence of disinfectants on them. Eastern-European journal of Enterprise Technologies, vol. 5, no. 11 p. 26-33. https://doi.org/10.15587/17294061.2017.110488

Langsrud, S., Moen, B., Møretrø, T. Løype, M., Heir, E. 2016. Microbial dynamics in mixed culture biofilms of bacteria surviving sanitation of conveyor belts in salmon processing plants. Journal of Apllied Microbiology, vol. 120, no. 2. p. 366-378. https://doi.org/10.1111/jam.13013

Merritt, K., Yuehuei, A. H. 2000. Factors Influencing Bacterial Adhesion. In Yuehuei, H. A., Friedman, R. J. Handbook of Bacterial Adhesion: Principles, Methods, and Applications. Totowa, New Jersey : Humana Press, p. 53-72. ISBN 978-1-61737-192-9. https://doi.org/10.1007/978-159259-224-1

Monds, R. D., O'Toole, G. A. 2009. The developmental model of microbial biofilms: ten years of a paradigm up for review. Trends in Microbiology, vol. 17, no. 2, p. 73-87. https://doi.org/10.1016/j.tim.2008.11.001

Moons, P., Michiels, C. W. 2009. Bacterial interactions in biofilms. Crit. Rev. Microbiol., vol. 35, no. 3, p. 157-168. https://doi.org/10.1080/10408410902809431

Moriarty, T. F., Poulsson, A. H. C., Rochford, E. T. J., Richards, R. G. 2011. Bacterial Adhesion and Biomaterial Surfaces, In: Ducheyne. Comprehensive Biomaterials. Oxford :ElsevierLtd., p. 75-100. https://doi.org/10.1016/B978-0-08$\underline{055294-1.00007-6}$

Nikolaev, B. A. 1976. Measuring of structural and mechanical properties of flour dough. Moscow : Pishchevaya Promyshlennost, $246 \mathrm{p}$.

Stadnyk, I., Novak, L., Matenchuk, L. 2018. Global rheological approach tothe quality of medium injected by the rollers. Potravinarstvo Slovak Journal for Food Sciences, vol. 12, no. 1, p. 397-404. https://doi.org/10.5219/867

Stadnyk, I., Piddubnuy, V., Eremeeva, O., Karpyk, H. 2018. Features of heat transfer in the environment when it is sprayed with rotary rollers.Potravinarstvo Slovak Journal of Food Sciences, vol. 12, no. 1, p. 824-835. https://doi.org/10.5219/977

Stadnyk, I., Piddubnyi, V., Karpyk, H., Kravchenko, M., Hidzhelitskyi, V. 2019. Adhesion effect on environment process injection. Potravinarstvo Slovak Journal for Food 
Sciences, vol. $13, \quad$ no. $\quad 1, \quad$ p. 429-437. https://doi.org/10.5219/1078

Stadnyk, I., Vitenko, T., Droździel, P., Derkach, A. 2016. Simulation of components mixing in order to determine rational parameters of working bodies. Advances in Science and Technology Research Journal, vol. 10, no. 31, p. 130138. https://doi.org/10.12913/22998624/64068

Whitehead, K. A., Verran, J. 2007. The Effect of Substratum Properties on the Survival of Attached Microorganisms on Inert Surfaces. In Flemming, H-C., Venkatesan, R., Murthy, P. S., Cooksey, K. Marine and Industrial Biofouling. Germany : Springer Series on Biofilms Springer,p. 13-33. ISBN 978-3-540-69794-7. https://doi.org/10.1007/978-3-540-69796-1_2

Zimon, A. D., Yevtushenko, A. M. 1985. Adgeziya pishhevyh mass (Adhesion of the food masses). Moscow, Russia : M. Agropromizdat, 272 p. (In Russian)

Zogaj, X., Bokranz, W., Nimtz, M., Rumling, U.2003. Production of cellulose and curli fimbriae by members of the family Enterobacteriaceaeisolated from the human gastrointestinal tract. Infect. Immun., vol. 71, no. 7, p. 41514158. https://doi.org/10.1128/IAI.71.7.4151-4158.2003

\section{Contact address:}

*Prof. Dr. Igor Stadnyk, Ternopil Ivan Puluj National Technical University, Department of Food Biotechnology and Chemistry, Ukraine, Ternopil 46001, Hohol str. 6, Tel.: +380975454829,
E-mail: igorstadnykk@gmail.com

ORCID: https://orcid.org/0000-0003-4126-3256

Prof. Dr. Volodymyr Piddubnyi, Kyiv National University of Trade and Economics, Faculty of Biotechnology and Food Sciences, Department of Technologies and Organization of Restaurant Business, Kyoto str. 19, Kyiv 02156, Ukraine, Tel.: +380674017096, E-mail: a.poddubnaya@i.ua

ORCID: https://orcid.org/0000-0001-8051-3743

Associate prof. Svitlana Krsnozhon, Kyiv National Economic University named after Vadym Hetman, Faculty of finance, Department of corporate finance and controlling, Prospect Peremogy 54/1, Kyiv, 03680. Ukraine, Tel.:+380502271147,

E-mail: svelte@ukr.net

ORCID: https://orcid.org/0000-0002-53676-4557

Antoshkova Nataliia, Khmelnitskyi cooperative trade and economic institute, Department of Hotel and Restaurant Business and Tourism, Kamianetska Str., 3 Khmelnitskyi 29000, Ukraine, Tel.:+ +380682676010,

E-mail: antoshkova209@ukr.net

ORCID: https://orcid.org/0000-0003-4715-8182

Corresponding author: * 